\title{
Selection of the distributional rule as an alternative tool to foster cooperation in a Public Good Game
}

\author{
Annarita Colasante \\ Università Politecnica delle Marche, Department of Economics and Social \\ Science. Piazzale Martelli, 8 Ancona (Italy) \\ e-mail: a.colasante@univpm.it
}

\begin{abstract}
This paper presents an investigation about cooperation in a Public Good Game using an Agent Based Model calibrated on experimental data. Starting from the experiment proposed in Colasante and Russo (2016), we analyze the dynamic of cooperation in a Public Good Game where agents receive an heterogeneous income and choose both the level of contribution and the distribution rule. The starting point is the calibration and the output validation of the model using the experimental results. Once tested the goodness of fit of the Agent Based Model, we run some policy experiment in order to verify how each distribution rule, i.e. equidistribution, proportional to contribution and progressive, affects the level of contribution in the simulated model. We find out that the share of cooperators decreases over time if we exogenously set the equidistribution rule. On the contrary, the share of cooperators converges to $100 \%$ if we impose the progressive rule. Finally, the most interesting result refers to the effect of the progressive rule. We observe that, in the case of high inequality, this rule is not able to reduce the heterogeneity of income.
\end{abstract}

Keywords: Public Good Game, Cooperation, Social Influence

\section{Introduction}

Cooperation among animal and human species has been widely studied from different perspectives ranging from social sciences to physics (see for example Mesterton-Gibbons and Dugatkin (1997)). Specifically, whereas physics mainly deals with the emergence of cooperation or segregation (see, for instance Helbing et al. (2011)), mainstream Economics suggests a selfish view of human actions, where agents maximize their own profit. A more recent literature both in Economics an Physics, however, shows that agents are not selfish (Kolm and Ythier (2006)), but often they are willing to cooperate also with strangers (Keser and Van Winden (2000), Andreoni and Croson (2008), Tedeschi et al. (2014), Vitali et al. (2013)). The wide literature in Experimental and Behavioral Economics try to understand the reason behind this behavior which seems to characterize human being. Using different kind of games such as Prisoner Dilemma, Ultimatum Game and Public Good Game, it has been shown that subjects do not behave as an homo œeconomicus but they are, on average, altruist in a bilateral game or they behave as an homo reciprocans in a multi-player setting (Szolnoki and Perc (2012), Tian et al. (2016), Fehr and Gächter (1998)). 
By employing a repeated Public Good Games (PGG hereinafter) it has been shown that cooperation, measured by the level of contribution to a public fund, even if decreases over time, always remains positive (Chaudhuri (2011)). Despite this evidence, there are many works which investigate different factors that could be foster the cooperation. Fist of all, the introduction of a punishment or a costly monitoring (Rustagi et al. (2010), Nikiforakis and Normann (2008)) or a reward (Szolnoki and Perc (2010b), Szolnoki and Perc (2013)) in the context of a PGG has a positive impact on cooperation. Agents, in fact, are willing to sustain a costly punishment to educate defectors in order to increase the level of cooperation and, so, their future benefits. A different approach to increase the contribution is to consider the voluntary participation (Semmann et al. (2003), Szolnoki and Chen (2015)), i.e. agents cooperate only if the number of defectors are less than a certain threshold (Szolnoki and Perc (2010a)). Another important factor that influences the cooperation is the group size (Szolnoki and Perc (2011), Isaac and Walker (1988)) and/or the interaction among agents (Szolnoki et al. (2009), Santos et al. (2008)), since there is not a clear effect of the size on contribution, i.e. not in every case we observe that the larger the group, the lower the contribution. Finally, there are also some works that explain the level of contribution as a reinforcement learning process (Nax and Perc (2015), Nax (2015)). For an exhaustive review on the different elements that are able to influence the contribution to the public good see Perc et al. (2013).

It is worth noting that, standard PGG just includes a simple control variable, namely the decision to contribute or not and, possibly, the amount of the contribution. An interesting exception is the game described by Colasante and Russo (2016). These authors present a PGG experiment with human subjects, where participants receive different initial endowments (or income) as in Tavoni et al. (2011) and Wang et al. (2010). Agents have not only to decide the amount of the contribution to the public fund, but also how to split the public good. Indeed, after all agents have chosen their amount of contribution, subjects in the same groups vote for the distribution rule among three feasible alternatives: $i$ ) equidistribution i.e. the public good is equally split among players independently on their contribution; ii) proportional to contribution i.e. the higher the contribution, the higher the share of the public good; iii) progressive i.e. agents with low endowment receive high share independently of their contribution.

According to the experimental evidence, the level of contribution is increasing over repetitions. This means that the possibility to decide how to split the public good is an alternative to costly punishment or reward to sustain cooperation.

This paper aims to shed light on the emergence of cooperation in the PGG experiment proposed by Colasante and Russo (2016). By developing an Agent-Based Model (ABM, hereinafter) reproducing the experimental setting, we analyze the evolution of cooperation along a dynamic path in terms of percentage of cooperators, and the impact of the three distribution rules in generating it. The robustness of the model in reproducing experimental data is then tested via the parameters estimation. Specifically, we show that the simulated time series generated by the agent-based model well reproduce the experimental data. Moreover, the calibration of the model parameters provides some information on the behavior of human subjects. In fact, the values of the parameters resulting from the model calibration show the differences and the similarities in the behavior of agents using different distribution rules.

There are many techniques to calibrate and validate ABMs. Janssen and Ostrom (2006) identify three useful environments in order to calibrate the model parameters: a) empirical data (see Recchioni et al. (2015) and Alfarano et al. (2007)), b) survey and/or interviews (Garcia et al. (2007)), c) experiments (Roth and Erev (1995)). Moreover, different validation techniques have been also proposed (see Sargent (2013)). Windrum et al. (2007), for instance, propose the so-called Werker-Brenner approach consisting in different phases. First, to identify the main stylized facts one wants to reproduce. Second, to include the use of the real data, empirical 
or experimental, to identify the most important parameters and/or the main behavioral rules. Finally, after obtaining results, to implement a validation exercise able to reproduce all the possible scenarios obtained by changing the initial conditions.

The process of validation and calibration of ABM with experimental data is one of the most innovative techniques. Experiments, both field and lab, are useful to observe directly the individual behavior in a controlled setting. Especially in the lab, it is possible to control all the variables involved except one, the control variable. This procedure allows to understand the effect of the control variable on the real choices. Moreover, experiments allow us to observe agents' interactions and, consequently, to derive aggregate properties which are the self-organized outcomes of the interactions of components (see for instance Tedeschi et al. (2012)).

In this paper, we follow the Werker-Brenner approach. Specifically, we firstly use experimental results and the behavioral rules highlighted in the empirical investigation of Colasante and Russo (2016) to calibrate the initial parameters of the model. Then, after checking the ability of the model to accurately match the experimental results, we change the value of the calibrated model parameters in order to identify the effect on the evolution of cooperation under the three different distributive rules. In particular, we check the effect of imposing a specific rule in all periods. The aim of these experiments is to understand how the cooperation, and so the contribution, evolves according to different distribution rules. Our finding shows that the higher level of cooperation is reached by applying the proportional rule. The most interesting results regards the relation between cooperation and inequality. Indeed, we find out that, by imposing the progressive rule in presence of high inequality, we observe a high level of cooperation but a low reduction of inequality. This result seems to be in contrast with the aim of the rule, i.e. reduce the initial level of inequality. The explanation behind this evidence relies on the low willingness to contribute of rich agents in the case of progressive rule.

The rest of the paper is organized as follows: in Section 2, we describe the ABM and in Section 3, we show the main results of the model both in terms of replication of the real data and the validation exercise. Finally, in Section 4, there are the conclusion and the final remarks.

\section{The model}

\subsection{Summary of the lab experiment}

In this Subsection we briefly describe the experiment proposed by Colasante and Russo (2016). Specifically, we present the key ingredients used to build the agent-based model which has to computationally reproduce the laboratory experiment. In the experiment, at the beginning of the round, each subject receives an endowment which can be used to contribute to a public fund. The experiment consists in comparing the individual behavior in terms of contribution to a public fund with different level of inequality. In fact, there are three different treatments: Treatment 1 with no inequality, Treatment 2 with a low level of inequality and Treatment 3 with high inequality. The difference among treatments are well explained in the next Subsection.

After receiving the endowment, each subject $i$ chooses the amount to put in the public fund $\left(g_{i t}\right)$. The contribution is continuous rather than binary as in Yuan and Xia (2014), Roberts and Sherratt (1998) and Zhang and Perc (2016). The difference between the endowment and the contribution constitutes a gain for the subject. The sum of the contribution of players in the same group constitutes the public good, i.e. the public good is given by

$$
G_{t}=\delta * \sum_{i=1}^{5} g_{i t}
$$


which $1<\delta<N$ represents the efficiency factor, i.e the synergetic effect of cooperation. In the experiment we set $\delta=2$. Usually, in the standard PGG, the public good is split equally among the components of the group. In the setting proposed by the authors, subjects decide how to split $G$ by voting for one of the three feasible rules: equidistribution, proportional and progressive. Each rule has a specific impact on the income distribution. In particular, we expect that the equidistribution rule leaves the same level of inequality, while the proportional rule is expected to raise heterogeneity and, finally, the progressive rule has the aim of reducing inequality. The rule receiving the majority of votes (i.e. at least 3 votes over 5) will be applied. At the end of each period the profit are computed. The profit function is:

$$
\pi_{i t}=d_{i t}-g_{i t}+\alpha_{i t} * G_{t}
$$

where $\alpha$ is the share of the public good gained. The value of $\alpha$ changes according to the chosen rule, i.e. the

- Equidistribution: $\alpha=\frac{1}{n}$;

- Proportional: $\alpha=\frac{g_{i}}{\sum_{i=1}^{5} g_{i}}$;

- Progressive: $\alpha=\frac{1}{n-1}\left(1-\frac{d_{i}}{\sum_{i=1}^{5} d_{i}}\right)$.

After the first period the endowment depends on the performance of the previous period, i.e. $d_{i t}=d_{i 0}+\alpha_{i t-1} * G_{t-1}$, where $d_{i 0}$ is the initial exogenous endowment. This implies that the new endowment includes also the share of the public good of the previous period, i.e. the we assume that the benefit of the public good does not end in the same period. This sequence of events is repeated for five periods. Throughout the whole game, subjects interact with the same people which are part of their own group.

\subsection{Implementation of ABM}

This Subsection describes all the micro mechanisms we implement in the ABM to replicate the aggregate experimental results regarding the contribution to the Public Good. In order to reproduce the observed behavior in the laboratory experiment, we build an ABM with four groups of five people each, i.e. $n=5$ is the number of people in each group and $N=20$ is total number of agents for each treatment. Each agent only interacts with its neighborhood, i.e. agents of the same group, for all the 5 periods $(T=5)$.

We consider three frameworks in which the initial distribution of the endowment changes to mimic the three experimental treatments. The treatments differ in the initial level of heterogeneity in the endowment distribution. In Treatment 1 (T1) all agents receive the same initial endowment $\left(d_{i 0}\right)$ equal to 100 . In Treatment 2 (T2) and Treatment 3 (T3) the distribution is such that the Gini index is equal to 0.2 and 0.6, respectively. Figure 1 shows the distributions of $d_{i 0}$ in T2 and T3. The distribution of the endowment within the group is common knowledge, this implies that each agent is aware of the level of inequality in its own group.

We calibrate the initial parameters using at the experimental data. The first parameter is the percentage of cooperators in the first period, i.e. $C_{T 1}=C_{T 1}^{0}, C_{T 2}=C_{T 2}^{0}$ and $C_{T 3}=C_{T 3}^{0}$. Since in the lab experiment the choice to contribute is not a binary choice, we define as cooperator those who contribute at least the $50 \%$ of their endowment. We split agents between Cooperators (C) or Defectors (D) according to this rule.

The percentage of cooperator is set as a parameter only in the first period. In the subsequent periods the decision to cooperate is endogenous and it depends on the rule chosen in the previous 

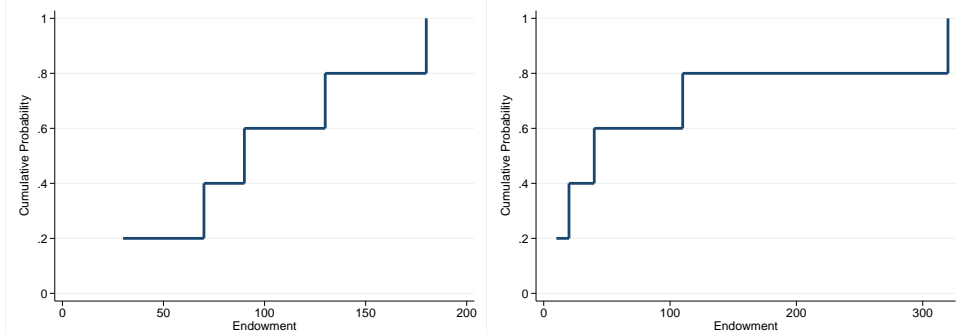

(a) Treatment $2(\mu=100, \sigma=52.13$,(b) Treatment $3(\mu=100, \sigma=117.09$, $\left.\gamma_{3}=0.24\right)$ $\left.\gamma_{3}=1.19\right)$

Figure 1: Distribution of of the endowment in Treatment 2 and Treatment 3.

period. For each of these rules, we choose simple mechanisms to define if agent $i$ belongs to the set of cooperators. In the following we describe for each rule, i.e. equidistribution, proportional to contribution or progressive to endowment, the main determinants in the decision to be a cooperator.

Regarding the Equidistribution rule, the theoretical predictions (see for example Fischbacher and Gächter (2010)) suggest that, in the standard PGG, the best individual strategy is to free ride, i.e. $g_{i}=0$. Moreover, in the experiment we observe that subjects behave as a conditional cooperators, meaning that they are willing to increase their contribution if they observe a cooperative behavior in the group. Considering these facts, we assume that the probability to cooperate to be a cooperator is, on the one hand, a function of the observed aggregate contribution and, on the other hand, it depends on the time. In particular, the probability to be a cooperator increases if the aggregate contribution of the previous period $(\nu)$ was greater than $40 \%$, and it decreases as the game approaches to the last period.

Regarding the Proportional rule, this guarantees each agent to receive back an amount greater than the contribution in any case. As described in Colasante and Russo (2016), this is the unique case in which the best choice for subject is to cooperate independently of the others' behavior. We implement rule of thumb based on the profit analysis; according to this rule, agent $i$ is a cooperator if $\left(\pi_{i t-1}-\pi_{i t-2}\right)>0$ and a defector otherwise, i.e. agents cooperate if they observe an increase in their own profit. Notice that, for the first period we must use a proxy of this difference because there is no value for $\pi_{i t-2}$. We consider as a proxy the difference between the the individual profit and the average profit of the group.

The decision to be a cooperator if the chosen rule in the previous period was the Progressive one depends on the individual endowment because the benefit $\alpha$ is independent on the contribution. Agents are split between rich and poor. Agents with an endowment higher than the average are defined as "rich", otherwise they are "poor". Since the progressive rule is in favor of the poor agents independently of their contributions, the probability to became a cooperator for the poor $\left(P(C)_{\text {poor }}\right)$ is higher than that for the rich $\left(P(C)_{\text {rich }}\right)$. We calibrate this probability looking at the percentage of cooperators in the experiment. According to the experimental data, we observe that, in many cases, the willingness to cooperate for rich agents decreases if the progressive rule is often chosen and the reverse holds for poor agents. Taking into account this feature, we assume that as the number of times the Progressive rule is chosen increases, $P(C)_{\text {poor }}$ rises and $P(C)_{\text {rich }}$ decreases.

In the empirical investigation proposed by Colasante and Russo (2016), authors find out that players show the so-called inequity aversion. This kind of behavior has a strong influence on 
the chosen rule. It has been shown, in fact, that the higher the level of inequality, the higher the frequency at which the progressive rule has been chosen. To take into account this aspect, we implement a mechanism of the distribution rules' choices depending on the Gini coefficient, i.e. a measure of inequality in the group. The rule is chosen according to a random selection from a normal distribution. The mean of the distribution depends on the degree of inequality in the group, i.e. the probability to choose a Progressive rule increases with inequality. The Gini coefficient is computed using the following equation ( $\mathrm{Xu}(2003))$ :

$$
\operatorname{Gini}_{t}=1-\frac{\sum_{i=1}^{n} f\left(d_{i}\right)\left(S_{i-1}+S_{i}\right)}{S_{n}}
$$

where $d_{i}$ are the endowment set in a not decreasing order and $S_{i}=\sum_{j=i}^{i} f\left(d_{j}\right) d_{j}$.

The robustness of our qualitative results has been checked by using Monte Carlo techniques. We have run 200 independent simulations for different values of the initial seed generating the pseudo-random numbers. All the initial parameters are based on the experimental ones. We use equations (1) and (2) to compute, respectively, the amount of the public good and the individual profit.

\section{Main results}

In this Section we show the main results of the simulation. To obtain our results we follow these steps: first, we make an exercise of output calibration starting from the real experimental data. The aim of this exercise is to check if the ABM is able to match the laboratory data. Second, we test the impact of each specific rule on cooperation. Specifically, we focus on the effect on both the contribution, and so the the share of cooperators, and distribution of the endowment for a given rule. We take a look also at the endowment distribution because, it has been shown in previous work like, that high inequality is detrimental for cooperation. In order to do so, we exogenously set the same rule in all periods, i.e. we run different computational experiments in which the distribution rule is equidistribution or proportional or progressive.

Let's start to analyze our calibration procedure. We set the value of the percentage of cooperators in each treatment equal to $C_{T 1}=40 \%, C_{T 2}=30 \%$ and $C_{T 3}=40 \%$. We also set the mean and standard deviation of the contribution in percentage term for both cooperators and defectors. The value of these parameters are shown in Table 1.

Table 1: Parameters of the model

\begin{tabular}{lccc}
\hline & T1 & T2 & T3 \\
\hline Cooperators & $\mu_{C}=0.6, \sigma_{C}=0.3$ & $\mu_{C}=0.55, \sigma_{C}=0.1$ & $\mu_{C}=0.55, \sigma_{C}=0.2$ \\
Defector & $\mu_{D}=0.3, \sigma_{D}=0.1$ & $\mu_{D}=0.3, \sigma_{D}=0.1$ & $\mu_{D}=0.25, \sigma_{D}=0.1$ \\
\hline
\end{tabular}

Following the output calibration procedure, after setting the value of the initial parameters and, by implementing the behavioral rules explained in the previous Section, we compare the main results of the model with the experimental data. In particular, we take into account the average contribution in percentage term, i.e. the average share of the endowment in each period for all the agents, and the chosen rule in each treatment.

As Figure 2 shows, the simulated time series are good in reproducing the average contribution observed in the experimental data. To test whether results emerging from the simulations are 
close to real data, we compare these results running a Kolmogorov-Smirnov test. In all the cases the test is not able to reject the null hypothesis at $5 \%$ conventional level of confidence (T1:pvalue $=0.207 ; \mathrm{T} 2: \mathrm{p}$-value $=0.456 ; \mathrm{T} 3: \mathrm{p}$-value $=0.182)$. We also run a Wilcoxon test. Also this test confirms the equality of the distributions $(\mathrm{T} 1: \mathrm{p}$-value $=0.345 ; \mathrm{T} 2$ :p-value $=0.138 ; \mathrm{T} 3$ : $\mathrm{p}$-value $=$ $0.685)$.

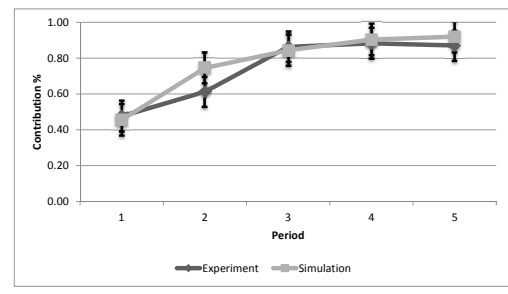

(a) Treatment $1($ Gini $=0)$

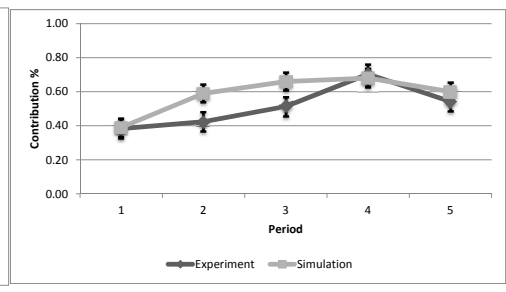

(b) Treatment $2($ Gini $=0.2)$

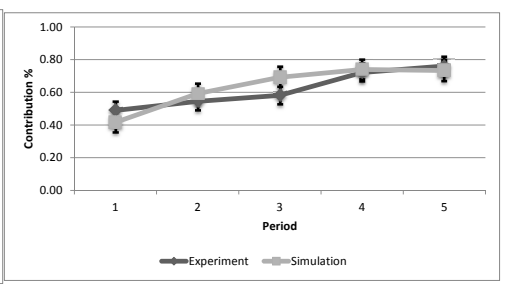

(c) Treatment $3($ Gini $=0.6)$

Figure 2: Each sub-graph compares the time series of the aggregate contribution in percentage of the endowment of simulated and experimental data. Grey squared lines reproduce simulated data. Black circle lines reproduce experimental data. The vertical lines represent the standard deviation of the variable. The simulated data well reproduces the experimental results.

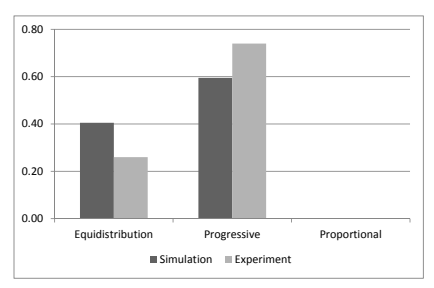

(a) Treatment $1($ Gini $=0)$

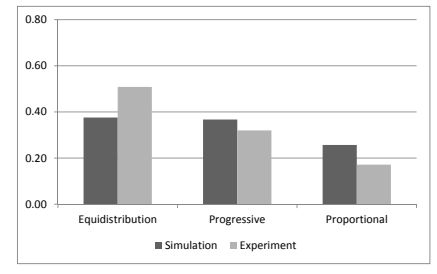

(b) Treatment $2($ Gini $=0.2)$

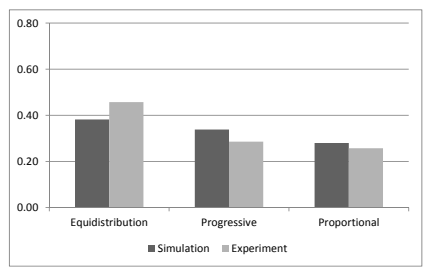

(c) Treatment $3($ Gini $=0.6)$

Figure 3: Comparison of simulated and real data regarding the percentage of the chosen rule in each Treatment. Grey bars indicate experimental data. Black bars indicate simulated data.

Figure 3 shows the frequency distribution for each rule in the different treatments. By using the rule based on the inequity aversion, the simulated results are very close to the real ones. Indeed, in the model relative to $\mathrm{T} 1$ the progressive rule has been never chosen in both the simulated and the real data. Our results confirm that the behavioral rules implemented for the choice of both the level of contribution and the distribution rule are able to replicate the behavior of agents during the experiment.

Once the real data are replicated, the model could be used to run some experiments. Specifically, we are interested in analyzing how imposing a specific rule affects subjects' cooperation. We want to test if, by changing the distributional rule, we observe a significant change in the cooperative behavior. Unlike what happens during the experiment in which players can choose one of the feasible rules, we are interested in understanding how the results change if we impose a specific rule. To observe these results we exogenously fix a specific rule, keeping unchanged 
other parameters. The aim of this experiment is to investigate if, and under which conditions, the contribution increases over time.

Figure 4 shows the aggregate contribution and the percentage of cooperators emerging in the model relative to $\mathrm{T}$ 1, i.e. the treatment in which there is no initial inequality. Note that we have omitted the results relative to the progressive rule because, in the case in which all agents receive the same endowment, the equidistribution and the progressive rule lead to the same results. By imposing the equidistribution rule in each period, both the average contribution and the percentage of cooperators decrease over time. This result is consistent with the evidence of the classic PGG where subjects have no incentive to cooperate if they are aware that the game is ending. On the other hand, if the proportional rule is chosen in all periods, all the agents become cooperators and they contribute with the whole endowment to the public good. The high level of cooperation reached with the proportional rule allows agents to accumulate a huge public good and, as a consequence, to increase their wealth in the subsequent periods. The disadvantage linked to this rule is that the inequality increases. As shown in Figure 5, the endowment is very high in the case of the proportional rule although the distribution is more heterogeneous.

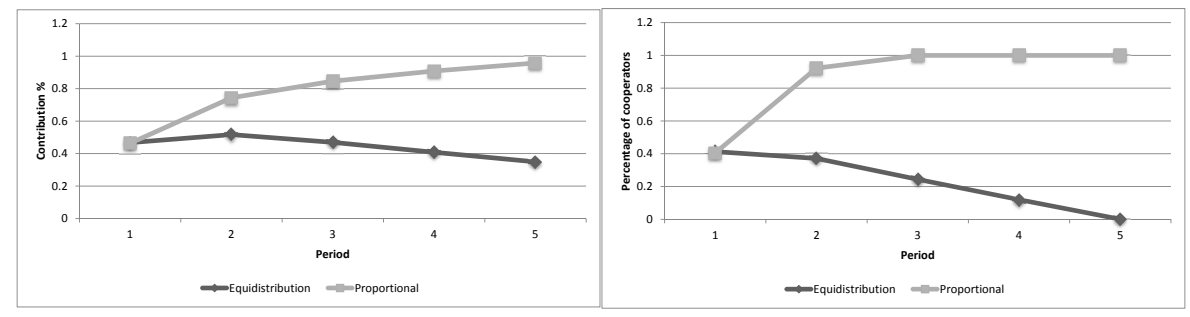

(a) Average contribution in percentage term.

(b) Percentage of cooperators.

Figure 4: Pannel (a) shows the average contribution relative to each rule in Treatment 1. Pannel (b) shows the percentage of cooperators in Treatment 1. In each pannel, the diamond black line refers to the equidistribution rule, the square gray line refers to proportional rule. By imposing the equidistribution rule, the percentage of cooperator approaches to zero at the end of the game, while, by imposing the proportional rule, all agents cooperate.

Figure 6 shows the average contribution to the public good and the share of cooperators in the model relative to T2. Also in this case, by imposing the equidistribution rule, the contribution declines over time and the share of cooperators goes to zero. Regarding the proportional rule, also in this setting there is a convergence to the full contribution. The imposition of the progressive rule results in a slightly decline of the share of cooperators during repetitions, while the average contribution remains fairly stable. The decline of cooperators is strongly influenced by the presence of "rich" agents. Indeed, half of agents holds an income above the mean and so they have no incentive to cooperate if the progressive rule is chosen for more than two periods. As shown in Figure 7, by imposing the proportional rule the inequality significantly increases, while, the progressive rule does not change the endowment distribution.

The same results emerge Figure 8, that is the highest contribution in the model relative to T3 corresponds to the proportional rule. The main difference is about the progressive rule. In this case, the percentage of cooperator remains close to the $80 \%$ and also the average contribution. This is because in this scenario the majority of players are "poor" and so they have the full incentive to cooperate. The impact of these different rules on the endowment distribution is very 


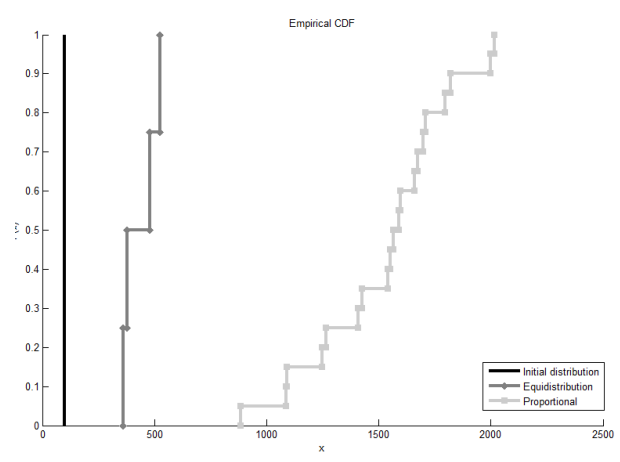

Figure 5: Cumulative Distribution of the endowment in the first and in the last period in Treatment 1. Diamond line represent the distribution we obtain by imposing the equidistribution rule. Squared line is the distribution regarding the proportional rule. The progressive rule increases the average income but increases the inequality as well.

different. As Figure 9 shows, the equidistribution rule leaves the distribution unchanged and increases the average endowment of players also in the case of high inequality. The proportional rule guarantees the high level of income but, at the same time, it increases the inequality and it benefits rich agents. The result regarding the progressive rule is the most interesting. If agents always choose this rule a double effect emerge. One the one hand, the degree of inequality decreases, i.e. in the case of $\mathrm{T} 3$ the inequality decreases by $20 \%$. This effect is the natural consequence of this rule, since it benefits the poor agents even if they are defectors. On the other hand, the growth rate of the endowment is very low and, even more important, the higher the inequality, the lower is the growth rate. These contrasting results lead to an important conclusion. The progressive rule increases the endowment of the poor players independently of their contribution and consequently reduces inequality. According to the results of the ABM, after 5 period the average endowment of poor agents increases by $400 \%$, while that of rich agents increases only by $7 \%$. Nevertheless, the gap between the highest and the lowest income is still very high until the last period. If the difference between poor and rich is very high -for example the case of T3-, it is very difficult to increase the level of contribution to the public good and so to have a strong reduction of the inequality. Indeed, the endowment of poor agents slowly increases during repetitions and so their "contributive capacity" remains too low to rise the amount of the public good. This implies that the public good remains, in all cases, very low and so the growth rate of the endowment is very slow.

Summing up, the main powerful tool to foster the cooperation is to guarantee a benefit proportional to the contribution even if, in the case of very high inequality, it does not guarantee the optimal result, meaning the full contribution to the public good.

\section{Conclusion}

In this work we present an agent based model calibrated using experimental data. The starting point is the experiment proposed in Colasante and Russo (2016) which is a PGG where players choose both the contribution and the distributive rule. Agents choose among three different rules: Equidistribution -the amount is equally split among all players-, Proportional to contribution -the share of public good is proportional to the contribution- and Progressive to endowment 


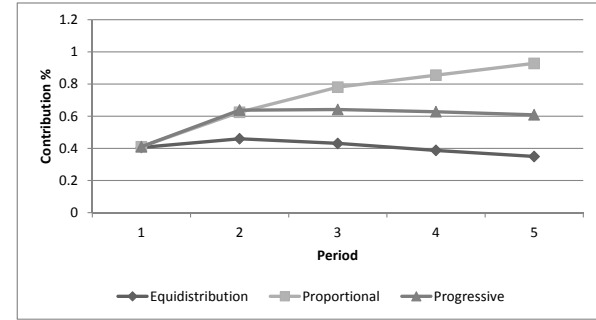

(a) Average contribution in percentage term.

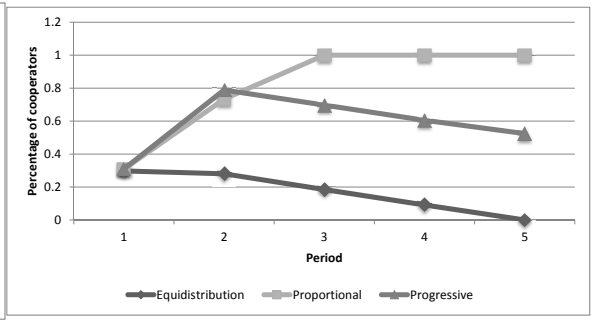

(b) Percentage of cooperators.

Figure 6: Pannel (a) shows the average contribution relative to each rule in Treatment 2. Pannel (b) shows the percentage of cooperators in Treatment 2. In each pannel, the diamond black line refers to the equidistribution rule, the square gray line refers to proportional rule and the triangle gray line refers to the progressive rule. The best performance in terms of cooperators and contributions, are achieved by imposing the proportional rule. The progressive rule sustains the cooperation better that the equidistribution one.

-the share of public good is inversely proportional to the endowment. Each of these rules has a different impact on the income distribution. We build a model based on three different frameworks, one for each treatments, i.e. $\mathrm{T} 1$ in which there is no initial inequality, $\mathrm{T} 2$ in which Gini coefficient is equal to 0.2 and T3 in which the Gini coefficient is equal to 0.6. The model is based on the experimental data and we implement behavioral rules deriving from the empirical investigation of Colasante and Russo (2016).

After testing the model ability in reproducing the experimental data via an output validation procedure, we run some policy experiments. Specifically, we analyze how the contribution and, in turn, the share of cooperators change according to different rules. To analyze this aspect we fix exogenously one rule for all repetitions. Results show that, by imposing the equidistribution rule, the share of cooperators decreases over repetitions in all treatments. The proportional rule leads to an increase of cooperators and, at the same time, a strong change in the income distribution. The most interesting result refers to the progressive rule. By imposing this rule for all the periods there is no clear effect on the income distribution. Especially considering the income distribution in T3, we observed that poor players increases their income but, the huge difference between rich and poor persists until the last round. This effect is peculiar since the main goal of the progressive rule should be to reduce the initial inequality.

A policy implication emerges from this analysis: in case of very high inequality, it is necessary to increase the endowment of the very poor agent in order to give them the possibility to increase their contribution to the public good. The joint effect of the progressive rule and the behavioral rule of rich agents, i.e. they reduce their contribution if the progressive rule is often chosen, is that there is no enough money in the public fund that could be used to reduce inequality.

The next step of the analysis will be to provide additional evidence about the importance of the contribution by rich players. The future investigation will focus on the comparison between a groups with and without rich cooperators. 


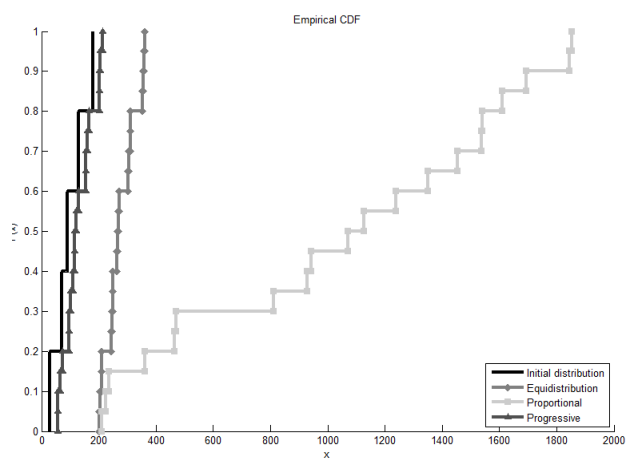

Figure 7: Cumulative Distribution of the endowment in the first and in the last period in Treatment 2. Diamond line represent the distribution we obtain by imposing the equidistribution rule. Squared line is the distribution regarding the proportional rule. The triangle line refers to the progressive rule. The proportional rule sharpen the inequality and the progressive rule have no strong impact on the income distribution.

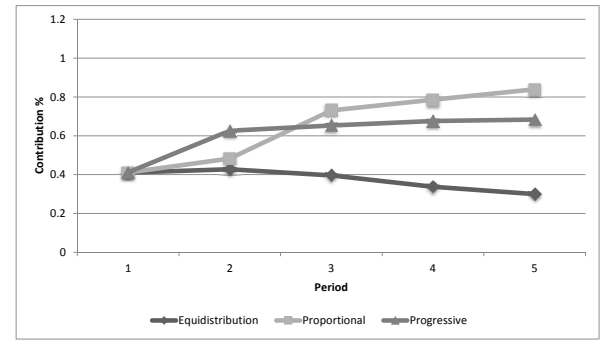

(a) Average contribution in percentage term.

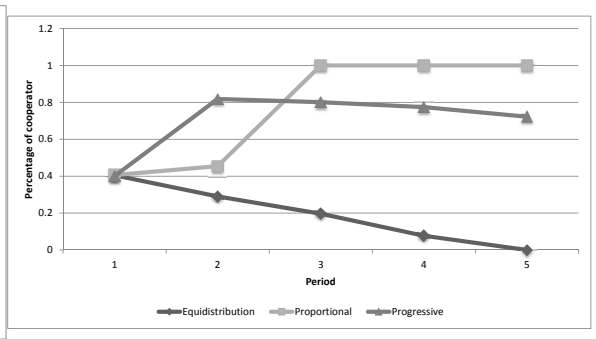

(b) Percentage of cooperators.

Figure 8: Pannel (a) shows the average contribution relative to each rule in Treatment 3. Pannel (b) shows the percentage of cooperators in Treatment 3. In each pannel, the diamond black line refers to the equidistribution rule, the square gray line refers to proportional rule and the triangle gray line refers to the progressive rule. In this case, the results in terms of contributions obtained by imposing the progressive rule are similar to that relative to the proportional one. 


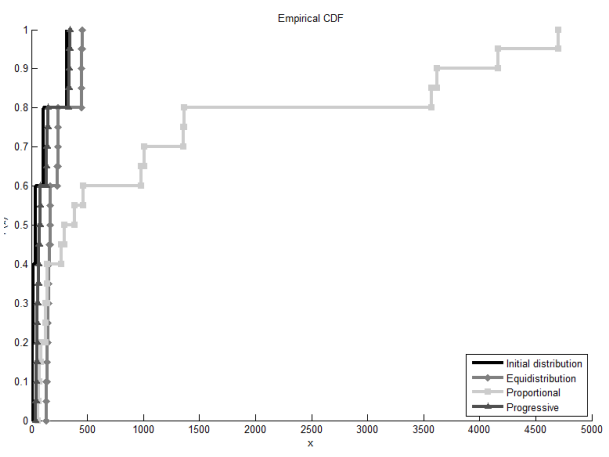

Figure 9: Cumulative Distribution of the endowment in the first and in the last period in Treatment 2. Diamond line represent the distribution we obtain by imposing the equidistribution rule. Squared line is the distribution regarding the proportional rule. The triangle line refers to the progressive rule. The proportional rule exacerbate the inequality by leaving the $40 \%$ of agents with a very low income. 


\section{Acknowledgement - Funding}

This research has received funding from the European Union, Seventh Framework Programme under grant agreements n. SYMPHONY- ICT-2013-611875.

\section{A Sensitivity Analysis}

\section{A.1 Analysis of the parameter $\nu$}

In this Section we check for the robustness of our results. First of all, we analyze how the results about cooperation change according to different value of the parameter $\nu$. This parameter represent the threshold of the aggregate contribution that determines if agent $i$ becomes a cooperator or not in the case of the equidistribution rule is chosen. We assign different values to $\nu$ in order to test if the results presented in the paper significantly change. As we said in Subsection 2.2, agent $i$ is a cooperator if $\nu>40 \%$. Now we consider four different values for $\nu$ : we choose two value close to our threshold, i.e. $30 \%$ and $50 \%$, and two extreme values, that is $10 \%$ and $90 \%$. We compare both the level of contribution and the share of cooperation for all these value of the parameter $\nu$.

In Figure 10 we show results relative to T1. In this case, since the equidistribution rule is chosen only in the $20 \%$ of times, there are no significant difference not in the contribution, nor in the percentage of cooperators. Indeed, the contribution remains fairly equal also if we consider the extreme case of a threshold equal to $90 \%$.

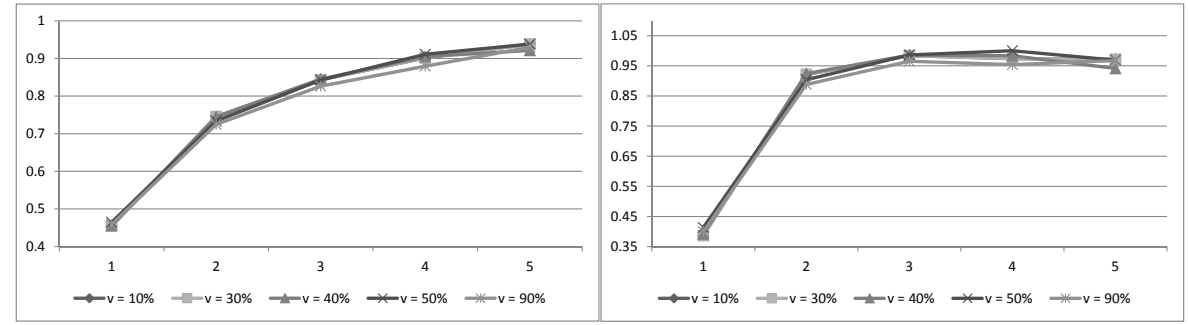

(a) Average contribution in percentage term.

(b) Percentage of cooperators.

Figure 10: Pannel (a) shows the average contribution relative Treatment 1. Pannel (b) shows the percentage of cooperators in Treatment 1. Each line corresponds to different value of the parameter $\nu$. There are no significant different not in term of cooperators, nor in terms of contributions.

Results relative to T2 are shown in Figure 11. In this scenario, there is no effect on both cooperators and contributors if we consider values close to the original threshold, i.e. $\nu=30 \%$ and $\nu=50 \%$. There is a reduction in the contribution only in the case in which we impose a very high limit equal to $\nu=90 \%$.

The results obtained for T3 are very similar to those for the T2 scenario. As we can see in Figure 12, the only significant effect on both contribution and cooperators are relative to a threshold equal to $\nu=90 \%$. 


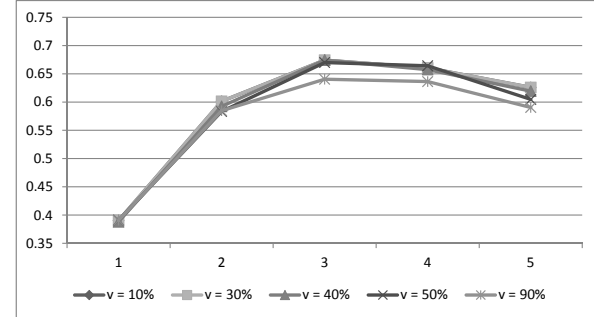

(a) Average contribution in percentage term.

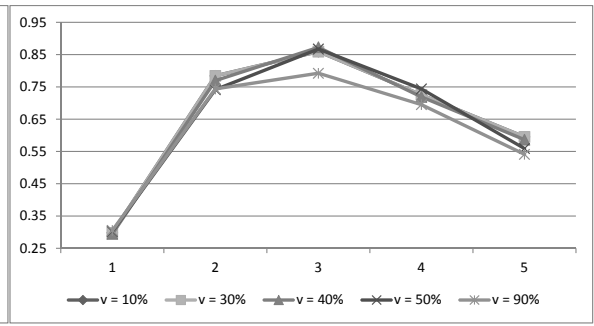

(b) Percentage of cooperators.

Figure 11: Pannel (a) shows the average contribution relative to Treatment 2. Pannel (b) shows the percentage of cooperators in Treatment 2. Each line corresponds to different value of the parameter $\nu$. We observe a significant reduction in both contribution and cooperators only if we fix $\nu=90 \%$.

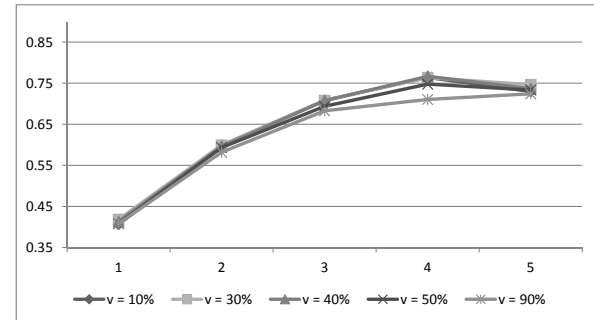

(a) Average contribution in percentage term

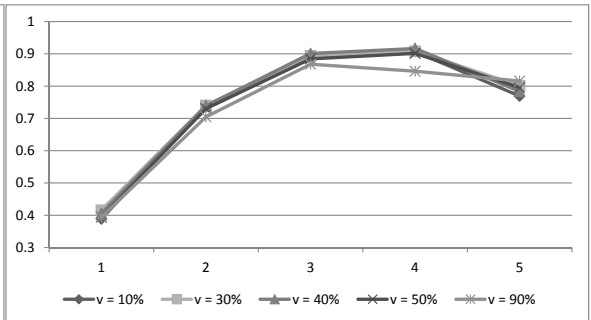

(b) Percentage of cooperators.

Figure 12: Pannel (a) shows the average contribution relative to Treatment 3. Pannel (b) shows the percentage of cooperators in Treatment 3. Each line corresponds to different value of the parameter $\nu$. We observe a significant reduction in both contribution and cooperators only if we fix $\nu=90 \%$. 


\section{A.2 Effect of a different time horizon}

The other interesting analysis regards the time horizon. In the simulation we consider as many periods as in the original experiment, i.e. $T=5$. In this Subsection we show how the results change if we extend the simulation to 20 periods. In the scenario relative to $\mathrm{T} 1$, the shape of the contribution is the same of the simulation. As we see in Figure 13, there is a fast convergence to the full contribution and, the main difference with respect to Figure 2(a) is that, starting from period 15 , the contribution slightly declines approaching to the $90 \%$.

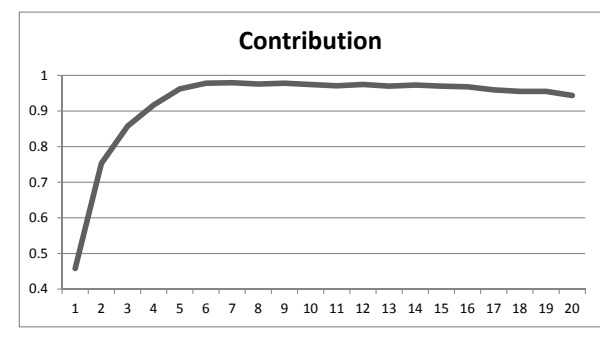

(a) Average contribution in percentage term.

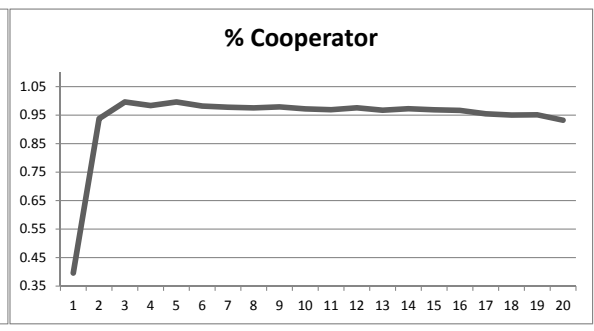

(b) Percentage of cooperators.

Figure 13: Pannel (a) shows the average contribution relative to Treatment 1 if we consider a $\mathrm{T}=20$. Pannel (b) shows the percentage of cooperators in Treatment 1.

In the scenario relative to T2, shown in Figure 14, the contribution grows up to reach $90 \%$ and, then, slowly decreases until the end of the simulation.

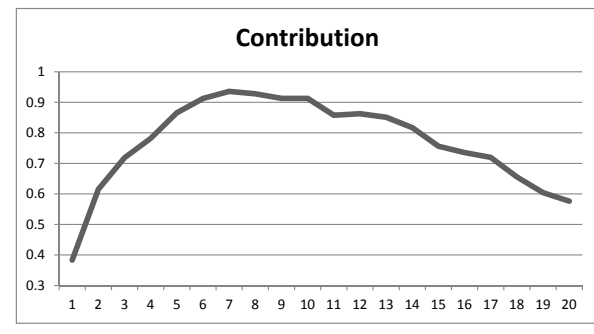

(a) Average contribution in percentage term.

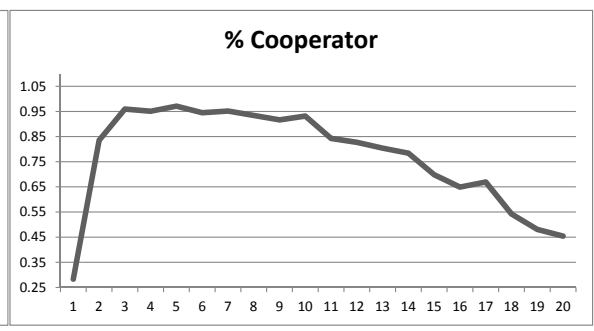

(b) Percentage of cooperators.

Figure 14: Pannel (a) shows the average contribution relative to Treatment 2 if we consider a $\mathrm{T}=20$. Pannel (b) shows the percentage of cooperators in Treatment 2.

In the last scenario, that relative to T3 and shown in Figure 15, we observe an inversion of the trend in the contribution. In fact, looking at Figure 2(c), the contribution is always increasing, but, by prolonging the time, there is a strong reduction in the contribution. This effects depends on the reluctance of rich agents to contribute to the public good if the progressive rule is often chosen. 


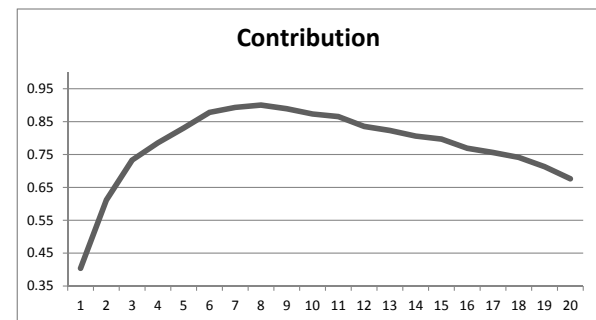

(a) Average contribution in percentage term

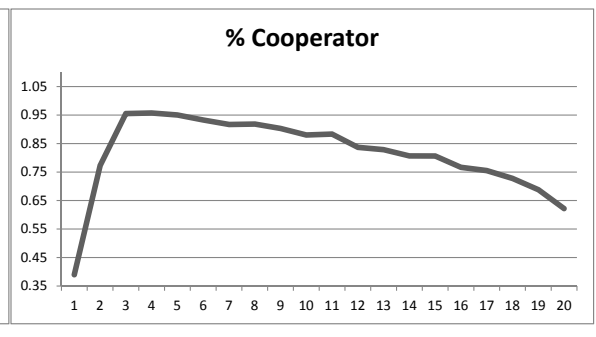

(b) Percentage of cooperators.

Figure 15: Pannel (a) shows the average contribution relative to Treatment 3 if we consider a $\mathrm{T}=20$. Pannel (b) shows the percentage of cooperators in Treatment 3. 


\section{References}

Alfarano, S., Lux, T., and Wagner, F. (2007). Empirical validation of stochastic models of interacting agents. The European Physical Journal B, 55(2), 183-187.

Andreoni, J. and Croson, R. (2008). Partners versus strangers: Random rematching in public goods experiments. Handbook of experimental economics results, 1, 776-783.

Chaudhuri, A. (2011). Sustaining cooperation in laboratory public goods experiments: a selective survey of the literature. Experimental Economics, 14(1), 47-83.

Colasante, A. and Russo, A. (2016). Voting for the distribution rule in a public good game with heterogeneous endowments. Journal of Economic Interaction and Coordination, pages 1-25.

Fehr, E. and Gächter, S. (1998). Reciprocity and economics: The economic implications of homo reciprocans. European economic review, 42(3), 845-859.

Fischbacher, U. and Gächter, S. (2010). Social preferences, beliefs, and the dynamics of free riding in public goods experiments. American Economic Review, 100(1), 541-556.

Garcia, R., Rummel, P., and Hauser, J. (2007). Validating agent-based marketing models through conjoint analysis. Journal of Business Research, 60(8), 848-857.

Helbing, D., Yu, W., and Rauhut, H. (2011). Self-organization and emergence in social systems: Modeling the coevolution of social environments and cooperative behavior. The Journal of Mathematical Sociology, 35(1-3), 177-208.

Isaac, R. M. and Walker, J. M. (1988). Group size effects in public goods provision: The voluntary contributions mechanism. The Quarterly Journal of Economics, pages 179-199.

Janssen, M. A. and Ostrom, E. (2006). Empirically based, agent-based models. Ecology and Society, 11(2), 37.

Keser, C. and Van Winden, F. (2000). Conditional cooperation and voluntary contributions to public goods. The Scandinavian Journal of Economics, 102(1), 23-39.

Kolm, S.-C. and Ythier, J. M. (2006). Handbook of the economics of giving, altruism and reciprocity: Foundations, volume 1. Elsevier.

Mesterton-Gibbons, M. and Dugatkin, L. A. (1997). Cooperation and the prisoner's dilemma: towards testable models of mutualism versus reciprocity. Animal behaviour, 54(3), 551-557.

Nax, H. H. (2015). Equity dynamics in bargaining without information exchange. Journal of Evolutionary Economics, 25(5), 1011-1026.

Nax, H. H. and Perc, M. (2015). Directional learning and the provisioning of public goods. Scientific reports, $\mathbf{5}$.

Nikiforakis, N. and Normann, H.-T. (2008). A comparative statics analysis of punishment in public-good experiments. Experimental Economics, 11(4), 358-369.

Perc, M., Gómez-Gardeñes, J., Szolnoki, A., Floría, L. M., and Moreno, Y. (2013). Evolutionary dynamics of group interactions on structured populations: a review. Journal of The Royal Society Interface, 10(80), 20120997. 
Recchioni, M. C., Tedeschi, G., and Gallegati, M. (2015). A calibration procedure for analyzing stock price dynamics in an agent-based framework. Journal of Economic Dynamics and Control, 60, 1-25.

Roberts, G. and Sherratt, T. N. (1998). Development of cooperative relationships through increasing investment. Nature, 394(6689), 175-179.

Roth, A. E. and Erev, I. (1995). Learning in extensive-form games: Experimental data and simple dynamic models in the intermediate term. Games and economic behavior, 8(1), 164-212.

Rustagi, D., Engel, S., and Kosfeld, M. (2010). Conditional cooperation and costly monitoring explain success in forest commons management. science, 330(6006), 961-965.

Santos, F. C., Santos, M. D., and Pacheco, J. M. (2008). Social diversity promotes the emergence of cooperation in public goods games. Nature, 454(7201), 213-216.

Sargent, R. G. (2013). Verification and validation of simulation models. Journal of Simulation, $7(1), 12-24$.

Semmann, D., Krambeck, H.-J., and Milinski, M. (2003). Volunteering leads to rock-paperscissors dynamics in a public goods game. Nature, 425(6956), 390-393.

Szolnoki, A. and Chen, X. (2015). Benefits of tolerance in public goods games. Physical Review E, 92(4), 042813.

Szolnoki, A. and Perc, M. (2010a). Impact of critical mass on the evolution of cooperation in spatial public goods games. Physical Review E, 81(5), 057101.

Szolnoki, A. and Perc, M. (2010b). Reward and cooperation in the spatial public goods game. EPL (Europhysics Letters), 92(3), 38003.

Szolnoki, A. and Perc, M. (2011). Group-size effects on the evolution of cooperation in the spatial public goods game. Physical Review E, 84(4), 047102.

Szolnoki, A. and Perc, M. (2012). Conditional strategies and the evolution of cooperation in spatial public goods games. Physical Review E, 85(2), 026104.

Szolnoki, A. and Perc, M. (2013). Correlation of positive and negative reciprocity fails to confer an evolutionary advantage: Phase transitions to elementary strategies. Physical Review $X$, 3(4), 041021.

Szolnoki, A., Perc, M., and Szabó, G. (2009). Topology-independent impact of noise on cooperation in spatial public goods games. Physical Review E, 80(5), 056109.

Tavoni, A., Dannenberg, A., Kallis, G., and Löschel, A. (2011). Inequality, communication, and the avoidance of disastrous climate change in a public goods game. Proceedings of the National Academy of Sciences, 108(29), 11825-11829.

Tedeschi, G., Gallegati, M., Mignot, S., and Vignes, A. (2012). Lost in transactions: The case of the boulogne s/mer fish market. Physica A: Statistical Mechanics and its Applications, 391(4), 1400-1407.

Tedeschi, G., Vitali, S., and Gallegati, M. (2014). The dynamic of innovation networks: a switching model on technological change. Journal of Evolutionary Economics, 24(4), 817-834. 
Tian, L.-L., Li, M.-C., and Wang, Z. (2016). Cooperation enhanced by indirect reciprocity in spatial prisoner's dilemma games for social p2p systems. Physica A: Statistical Mechanics and its Applications, 462, 1252-1260.

Vitali, S., Tedeschi, G., and Gallegati, M. (2013). The impact of classes of innovators on technology, financial fragility, and economic growth. Industrial and Corporate Change, 22(4), 1069-1091.

Wang, J., Fu, F., and Wang, L. (2010). Effects of heterogeneous wealth distribution on public cooperation with collective risk. Physical Review E, 82(1), 016102.

Windrum, P., Fagiolo, G., and Moneta, A. (2007). Empirical validation of agent-based models: Alternatives and prospects. Journal of Artificial Societies and Social Simulation, 10(2), 8.

Xu, K. (2003). How has the literature on gini's index evolved in the past 80 years? Dalhousie University, Economics Working Paper.

Yuan, W.-J. and Xia, C.-Y. (2014). Role of investment heterogeneity in the cooperation on spatial public goods game. PloS one, 9(3), e91012.

Zhang, H. and Perc, M. (2016). Evolution of conditional cooperation under multilevel selection. Scientific reports, 6. 